\title{
Impact of urinary incontinence on health-related quality of life, daily activities, and healthcare resource utilization in patients with neurogenic detrusor overactivity
}

Derek H Tang ${ }^{1 *}$, Danielle Colayco ${ }^{2}$, James Piercy ${ }^{3}$, Vaishali Patel ${ }^{2}$, Denise Globe ${ }^{2}$ and Michael B Chancellor ${ }^{4}$

\begin{abstract}
Background: Neurogenic detrusor overactivity (NDO) leads to impaired health-related quality of life (HRQoL), productivity, and greater healthcare resource burden. The humanistic and economic burden may be more apparent in NDO patients with urinary incontinence (UI). The objective of this study was to compare the HRQoL, productivity, and health resource use (HRU) between continent and incontinent NDO patients.

Methods: A retrospective database analysis was conducted using the Adelphi Overactive Bladder (OAB)/UI Disease Specific Programme, a multi-national, cross-sectional survey reported from both patients' and physicians' perspectives. The population for this analysis included NDO patients with or without UI. General and disease-specific HRQoL were assessed using the EuroQoL-5D (EQ-5D), Incontinence Quality of Life questionnaire (I-QOL), and the Overactive Bladder Questionnaire (OAB-q). Productivity and daily activity impairment were measured using the Work Productivity and Activity Impairment (WPAI) questionnaire. HRU indicators included OAB-related surgery, OAB-related hospitalizations, incontinence pad usage, switching anticholinergics used for $O A B$ due to inadequate response or adverse effects, and OAB-related physician visits. Bivariate analyses, multivariate ordinary least squares (OLS) regression analyses and published minimal clinically important differences (MCID) were used to assess relationships between incontinent status and the aforementioned outcome measures.
\end{abstract}

Results: A total of 324 NDO patients with or without urinary incontinence were included, averaging 54 years of age (SD 16), of whom 43.8 percent were male. Bivariate analyses detected no significant relationship between incontinent status and HRU variables. Regression analyses revealed that incontinent patients had clinically and statistically lower disease-specific HRQoL and greater impairment in daily activities as compared to continent patients. On average, incontinent patients scored 10 points lower on the I-QOL total score, 9 points lower on the OAB-q HRQoL score, 15 points higher on OAB-q symptom severity, and experienced 8.2 percent higher activity impairment due to their bladder condition (all $p<0.001$ ).

Conclusions: Incontinent NDO patients experience significantly lower HRQOL and activity impairment as compared to continent NDO patients.

Keywords: Neurogenic detrusor overactivity, Incontinence, Burden of illness, Quality of life, Productivity

\footnotetext{
* Correspondence: dtang@pharmacy.arizona.edu

${ }^{1}$ The University of Arizona College of Pharmacy, 1295 N. Martin, PO Box 210202, Tucson, AZ 85721, USA

Full list of author information is available at the end of the article
} 


\section{Background}

Overactive bladder $(\mathrm{OAB})$ has been defined by the International Continence Society as urgency with or without urinary incontinence (UI), usually with frequency and nocturia [1]. Compared with patients without OAB, those with $O A B$ suffer from lower health-related quality of life (HRQoL), higher prevalence of chronic comorbidities, and substantial economic burden. Total costs were proportional to the country population size; accounting for an estimated 65.7 billion USD in the US and 4.2 billion Euros in the five largest western European countries [2,3]. In addition, $\mathrm{OAB}$ patients were found less likely to be employed and had significantly worse general HRQoL (EQ-5D score) as compared to patients without OAB $[4,5]$.

Neurogenic detrusor overactivity (NDO) is defined as bladder overactivity due to a relevant neurological condition, such as multiple sclerosis (MS), spinal cord injury (SCI), Parkinson's disease (PD), or stroke [1]. The mainstay of therapy for NDO includes the use of anticholinergic medications, along with supportive care via behavioral training, clean intermittent catheterization and the use of absorbent pads [6,7]. Other treatment options include alpha-blockers, botulinum toxin, and surgery (augmentation cystoplasty).

While the economic and HRQoL burden have been estimated in the general OAB population, the burden attributable to UI in NDO patients has not been investigated $[4,8-10]$. Patients with a neurogenic condition may differ in their underlying comorbidities, types of specialists accessed for care, treatment choices, and ultimately, their treatment outcomes. Thus, the objective of the current study is to assess the impact of UI on patient HRQoL, productivity, and healthcare resource use in NDO patients.

\section{Methods}

This analysis was completed using an existing data set, the Adelphi OAB/UI Disease Specific Programmes (DSP), a cross-sectional survey, fielded between: November 2010 till February 2011. The Adelphi OAB/UI survey elicits patient-level data from the US, UK, France, Spain, and Germany, including patient demographics, physician practice trends, healthcare utilization, HRQoL, and productivity [11].

During the data collection phase, physicians were recruited via the use of publicly available lists of healthcare professionals for identification purposes, followed by a list of prespecified criteria for screening purposes (Table 1). Approximately 700 physicians completed a Patient Record Form (PRF) which provided information relating to patient demographics, employment status, bladder symptoms, disease severity, compliance, current and previous medications, and hospitalization data for the 10 to 12 most recent clinical visits for patients fulfilling the inclusion criteria over a two to three-week timeframe. Patients selected for chart abstraction and who agreed to participate completed a one-time, cross-sectional survey (i.e., the Patient Self-Completion [PSC] form) assessing $\mathrm{OAB}$ symptoms and their impact on daily functioning, HRQoL and work productivity. Due to the low prevalence of neurogenic bladder patients, each physician was asked to record data from at least two neurogenic patients to ensure sufficient representation in the convenience sample. Of the 7,430 charts abstracted, 4,027 (54.2\%) patients completed the survey. As no patient or physicianidentifiable information had been recorded within the data set (i.e., completely de-identified), no human subject approval is required.

Patient responses to a series of validated instruments were used to evaluate HRQoL and productivity level. General HRQoL was evaluated using the EuroQoL-5D 3 level (EQ-5D $3 \mathrm{~L}$ ) utility score [12]. EQ-5D is a standardized instrument providing a simple, generic measure of health, consisting of questions measuring five dimensions of health states (mobility, self-care, usual activities, pain/discomfort, and anxiety/depression) and a visual analog scale (VAS) measuring overall health. Utility score computation was derived from responses to all questions excluding the VAS. Disease-specific HRQoL was elicited using the Incontinence Quality of Life questionnaire (I-QOL) total score and the Overactive

Table 1 Inclusion criteria for physicians and patients to be included in the overall Adelphi OAB/UI survey*

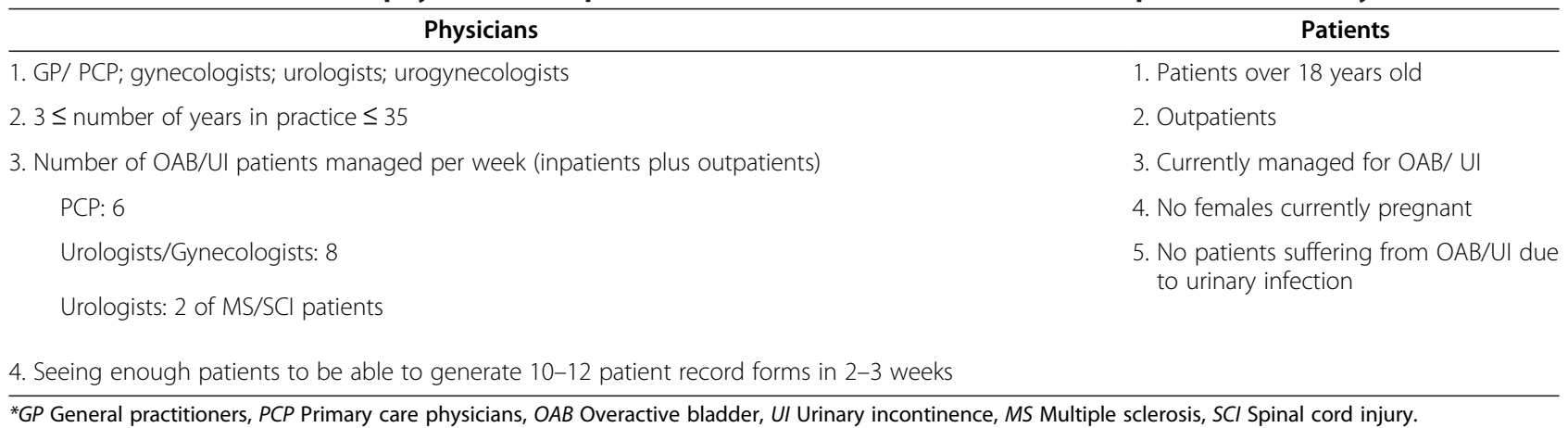

${ }^{*} G P$ General practitioners, $P C P$ Primary care physicians, OAB Overactive bladder, UI Urinary incontinence, MS Multiple sclerosis, SCI Spinal cord injury. 
Bladder Questionnaire (OAB-q) symptom severity score and HRQoL score [13,14]. I-QOL and OAB-q are 22-item and 33-item standardized instruments assessing the effect of urinary incontinence and symptom bother/HRQoL impact of OAB on patients' life, respectively. Finally, productivity was assessed using the Work Productivity and Activity Impairment (WPAI) questionnaire [15]. WPAI evaluates percent daily activity impairment, percent impairment while working (i.e., presenteeism), percent work time missed (i.e., absenteeism), and percent overall work impairment (i.e., combination of absenteeism and presenteeism). The WPAI questionnaire was adapted to $\mathrm{OAB} / \mathrm{UI}$ patients by specifically reflecting the impact of $\mathrm{OAB} / \mathrm{UI}$ on work productivity and daily activity (e.g., "During the past seven days, how many hours did you miss from work because of your bladder symptoms?"). Published scoring algorithms were used to generate total and domain scores for each measure [16-19]. The US algorithm was used to compute utility scores for the analytic sample because: 1) the possible range of the mapped score is a balance between using the UK algorithm and other utility deriving instruments (e.g., Health Utility Index II, III, and the Short Form-36), and 2) utility scores could be consistently estimated across international patients. Table 2 lists the possible score ranges and interpretation for each measure.

Health resource utilization was evaluated using the following measures: 1 ) proportion of patients that ever had OAB-related surgery; 2) proportion of patients having OAB-related hospitalization over the past 12 months; 3) proportion of patients currently using incontinence pads (at the time of completing the survey); 4) number of pads used per week; 5) proportion of patients that had switched anticholinergics used for $\mathrm{OAB}$ due to inadequate response or adverse drug reactions; and 6) number of OAB-related physician visits over the past 3 months. Hospitalizations, surgery, and anticholinergic history were derived from chart review, while the remainder of items was patient-reported.

\section{Patient selection}

To be included in the specific analytic sample for this research, patient data had to: (1) include an $\mathrm{OAB}$ diagnosis with or without incontinence associated with an underlying neurological condition; (2) have completed the PSC form; and (3) have a history of anticholinergics use for OAB. There has been limited focus on HRQoL and healthcare resource use in $\mathrm{OAB}$ patients exposed to more treatment options (i.e., whose disease condition has not been appropriately controlled with anticholinergics). Hence, we targeted this specific population to provide public data for researchers to use in future studies. Data from patients with functional, flow, stress, or mixed-type incontinence were excluded. Incontinence status (yes/no) was determined from a question on the PSC form, "Are there times when you experience leakages, however small the amount?" The frequency of incontinent episodes was obtained from the question, "Thinking about the last week... On average, what is the total number of leakages you experienced over a 24 hour period?" Only those patients reporting leakages were eligible to report the number of episodes in the second question.

\section{Statistical analysis}

First, Chi-square tests or Fisher's exact tests were used for categorical variables, while Student's t-tests or Wilcoxon rank-sum tests were used for continuous variables to explore differences between continent and incontinent patients in terms of HRQoL, productivity measures, and healthcare resource utilization to assess the relationship between incontinence and these factors. To further control for confounders that may potentially bias the relationship between incontinent status and health outcomes, multivariate ordinary least square (OLS) regression models were employed to evaluate associations between number of incontinent episodes and quality of life/productivity loss related to $\mathrm{OAB} / \mathrm{UI}$. Other secondary independent variables (including time since diagnosis, age,

Table 2 Instrument score range and interpretation ${ }^{\mathrm{a}, \mathrm{b}}$

\begin{tabular}{llcl}
\hline & \multicolumn{1}{c}{ Outcome measures } & Score range & Interpretation \\
\hline General Health Utility & EQ-5D 3 L utility score & $-0.11-1$ & General health $\uparrow$ as score $\uparrow$ \\
Disease-specific QOL & I-QOL total score & $0-100$ & HRQoL $\uparrow$ as score $\uparrow$ \\
& OAB-q symptom severity score & $0-100$ & Symptom severity $\downarrow$ as score $\uparrow$ \\
OAB-q HRQoL score & $0-100$ & HRQoL $\uparrow$ as score $\uparrow$ \\
Productivity & \% activity impairment due to their bladder condition & $0-100$ & Productivity $\downarrow$ as score $\uparrow$ \\
& \% work time missed due to their bladder condition & $0-100$ & Productivity $\downarrow$ as score $\uparrow$ \\
& $\%$ impairment while working due to their bladder condition & $0-100$ & Productivity $\downarrow$ as score $\uparrow$ \\
& $\%$ overall work impairment due to their bladder condition & $0-100$ & Productivity $\downarrow$ as score $\uparrow$ \\
\hline
\end{tabular}

${ }^{a} Q O L$, Quality of life; EQ-5D, EuroQoL-5D; I-QOL, Incontinence Quality of Life questionnaire; $O A B-q$, Overactive Bladder questionnaire; HRQoL, Health-related quality of life.

${ }^{\mathrm{b}} \downarrow$ indicates decrease, $\uparrow$ indicates increase.

'Derived from the 5 questions representing 5 dimensions of health states in the EQ-5D instrument using standardized scoring algorithm. 


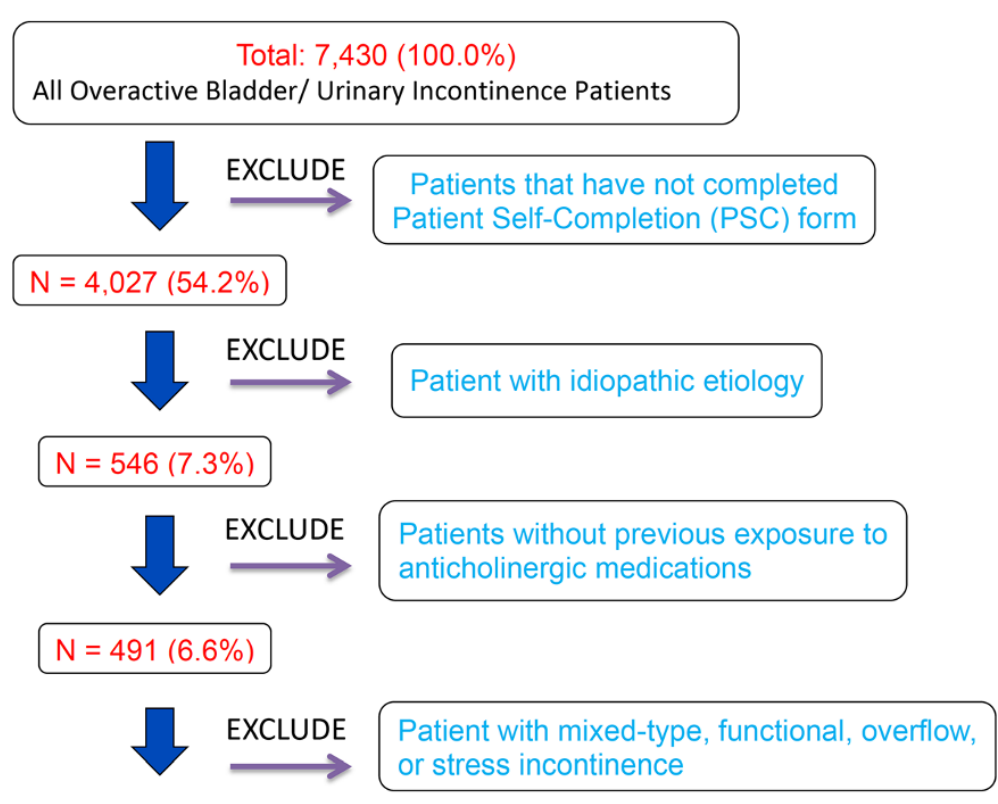

Final Analytic Sample N = 324 (4.4\%)

Figure 1 Patient cohort selection flowchart.

sex, race, education, insurance status, and comorbidities) used in the regression analyses were selected based on: 1) univariate regression analyses and descriptive statistics; 2) theoretically sound constructs possibly predictive of the endpoints; and 3) variables available in the survey.

The magnitude of score changes reported in the literature were used to assess whether effect sizes reached or exceeded the minimal clinically important differences (MCID) for each measure: EQ-5D utility score-0.03 to 0.07 [20,21]; I-QOL subscales-4 to 11 [13]; OAB-q HRQoL score-5 [22,23]; OAB-q symptom severity score-10 [22,23]. MCIDs for WPAI have been validated only in patients with insomnia and Crohn's disease $[24,25]$, and thus were deemed insufficiently reliable to be used for comparisons.
To test the robustness of these associations, sensitivity analyses were conducted on the incontinence variable by categorizing it as ordinal (continent; incontinent with 0 to 4 daily episodes; incontinent with more than 4 daily episodes) and continuous (number of UI episodes per week). Additional sensitivity analyses were also conducted using negative binomial models in lieu of OLS regression models. To minimize sample size loss due to missing data, median (for number of pads used and number of daily incontinent episodes) and multiple imputations (for all other study variables with missing data) were employed to predict values for missing data with respect to variables used in the regression and sensitivity analyses. Median imputation was used for the two variables because their values

Table 3 Distribution of underlying neurological conditions among continent and incontinent NDO patients ${ }^{\mathrm{a}-\mathrm{c}}$

\begin{tabular}{lcc}
\hline \multicolumn{1}{c}{$\begin{array}{c}\text { Neurological } \\
\text { conditions }\end{array}$} & $\begin{array}{c}\text { Continent } \\
\text { (n (\% all continent patients)) }\end{array}$ & $\begin{array}{c}\text { Incontinent } \\
\text { (n (\% all incontinent patients)) }\end{array}$ \\
\hline Parkinson's Disease (PD) & $6(7.7 \%)$ & $23(9.8 \%)$ \\
Multiple Sclerosis (MS) & $43(55.1 \%)$ & $122(51.9 \%)$ \\
Spinal Cord Injury (SCl) & $14(17.9 \%)$ & $52(22.1 \%)$ \\
Stroke & $10(12.8 \%)$ & $21(8.9 \%)$ \\
Other & $4(5.1 \%)$ & $12(5.1 \%)$ \\
Unknown & $1(1.3 \%)$ & $5(2.1 \%)$ \\
Total & $78(100.0 \%)$ & $235(100.0 \%)$ \\
\hline
\end{tabular}

${ }^{\mathrm{a}} 301$ (92.9\%) out of 324 NDO patients had a primary neurological condition of either PD, MS, SCl or stroke.

${ }^{\mathrm{b}} 11$ patients out of the 324 patients had unknown incontinence status.

${ }^{\mathrm{C}}$ Among all SCl patients, $29 \%$ had complete $\mathrm{SCl}, 61 \%$ had incomplete $\mathrm{SCl}$, while $10 \%$ had unknown injury status. 
Table 4 Patient demographics by incontinence status ${ }^{a}$

\begin{tabular}{|c|c|c|c|c|}
\hline & \multirow[t]{2}{*}{ Variables } & & \multicolumn{2}{|c|}{ Incontinence status $^{\mathrm{b}}$} \\
\hline & & & $\begin{array}{c}\text { Continent } \\
(\mathrm{n}=78)\end{array}$ & $\begin{array}{l}\text { Incontinent } \\
(n=235)\end{array}$ \\
\hline \multirow[t]{3}{*}{ Age (mean (sd)) } & USA & & $55.8(17.4)$ & $54.9(15.9)$ \\
\hline & Europe & & $49.9(16.5)$ & $54.1(16.2)$ \\
\hline & Total & & $51.5(16.9)$ & $54.3(16.1)$ \\
\hline \multirow[t]{3}{*}{ Male (n (\%)) } & USA & & $11(52.4 \%)$ & $23(35.4 \%)$ \\
\hline & Europe & & $24(42.1 \%)$ & 79 (47.0\%) \\
\hline & Total & & $35(44.9 \%)$ & $102(43.8 \%)$ \\
\hline Diagnosed $\geq 1$ year ago & USA $A^{* *}$ & & $9(52.9 \%)$ & $55(88.7 \%)$ \\
\hline \multirow[t]{2}{*}{ (n (\%)) } & Europe & & $38(73.1 \%)$ & $110(68.8 \%)$ \\
\hline & Total & & $47(68.1 \%)$ & $165(74.3 \%)$ \\
\hline \multirow[t]{12}{*}{ Ethnicity (n (\%)) } & USA & White/Caucasian & $11(52.4 \%)$ & $45(69.2 \%)$ \\
\hline & & Hispanic/Latino & $4(19.0 \%)$ & $5(7.7 \%)$ \\
\hline & & Afro/Caribbean & $5(23.8 \%)$ & $13(20.0 \%)$ \\
\hline & & Others & $1(4.8 \%)$ & $2(3.1 \%)$ \\
\hline & Europe & White/Caucasian & $48(84.2 \%)$ & $148(87.1 \%)$ \\
\hline & & Hispanic/Latino & $7(12.3 \%)$ & $9(5.3 \%)$ \\
\hline & & Afro/Caribbean & $2(3.5 \%)$ & $7(4.1 \%)$ \\
\hline & & Others & $0(0.0 \%)$ & $6(3.5 \%)$ \\
\hline & Total & White/Caucasian & $59(75.6 \%)$ & $193(82.1 \%)$ \\
\hline & & Hispanic/Latino & $11(14.1 \%)$ & $14(6.0 \%)$ \\
\hline & & Afro/Caribbean & $7(9.0 \%)$ & $20(8.5 \%)$ \\
\hline & & Others & $1(1.3 \%)$ & $8(3.4 \%)$ \\
\hline \multirow[t]{9}{*}{ Education (n (\%)) } & USA & Less than high school & $1(5.0 \%)$ & $1(1.8 \%)$ \\
\hline & & High school & $6(30.0 \%)$ & $22(39.3 \%)$ \\
\hline & & College or more & $13(65.0 \%)$ & $33(58.9 \%)$ \\
\hline & Europe & Less than high school & $6(11.1 \%)$ & $25(15.5 \%)$ \\
\hline & & High school & $18(33.3 \%)$ & $65(40.4 \%)$ \\
\hline & & College or more & $30(55.6 \%)$ & $71(44.1 \%)$ \\
\hline & Total & Less than high school & $7(9.5 \%)$ & $26(12.0 \%)$ \\
\hline & & High school & $24(32.4 \%)$ & $87(40.1 \%)$ \\
\hline & & College or more & $43(58.1 \%)$ & $104(47.9 \%)$ \\
\hline \multirow[t]{3}{*}{ Have any insurance (n (\%)) } & USA & & $18(100.0 \%)$ & $55(98.2 \%)$ \\
\hline & Europe & & $43(76.8 \%)$ & $125(76.7 \%)$ \\
\hline & Total & & $61(82.4 \%)$ & $180(82.2 \%)$ \\
\hline
\end{tabular}

aivariate analyses comparing distribution of demographic variables between incontinent versus continent group.

${ }^{\mathrm{b}} 11$ out of 324 NDO patients had unknown incontinent status.

${ }^{*} p<0.05{ }^{* *} p<0.01{ }^{* * *} p<0.001$ in chi-square test, Fisher's exact test, t-test, or Wilcoxon rank-sum test as appropriate.

were conditional on whether they had used any pads/had any incontinent episodes; missing data from both of the dichotomous variables were replaced using multiple imputation. SAS version 9.2 (Cary, NC) and STATA version 12.1 (College Station, TX) were used for data management and analysis, respectively. Significance level was set at $\mathrm{p}<0.05$.

\section{Results}

The final analytic sample included data from 324 patients (54.1\% of 599 patients completed the PSC form) (Figure 1). Compared with PSC responders, PSC non-responders were on average older (56.1 vs. 53.0, $\mathrm{p}=0.02)$; consisted of more White/Caucasian (83.1\% vs. $80.9 \%)$, minority races $(7.0 \%$ vs. $2.8 \%)$, and less 
Hispanic/Latino ( $1.1 \%$ vs. $8.0 \%) \quad$ (pethnicity <0.001); had more patients with a underlying neurological diagnosis of PD (15.6\% vs. 9.3\%) and stroke $(13.1 \%$ vs. 9.6\%), and less patients with MS $(42.2 \%$ vs. $52.8 \%)$ and SCI $(16.4 \%$ vs. $21.3 \%)$ ( $\left.\mathrm{p}_{\text {neuro }}=0.003\right)$. However, the effect size of these differences was not clinically meaningful. Time since diagnosis was comparable ( $\mathrm{p}=0.24$ ) between responders and non-responders. Patients from the US and Western European countries accounted for $27.5 \%(n=89)$ and $72.5 \%(n=235)$ of the final sample, respectively. The study sample averaged 53.5 years of age and consisted of $56.2 \%$ female, $80.9 \%$
non-Hispanic whites, and $75.1 \%$ incontinent patients. A primary neurological diagnosis of $\mathrm{PD}, \mathrm{MS}, \mathrm{SCI}$, or stroke was identified in $92.9 \%$ of the patients, with MS patients comprising over half of the sample (Table 3).

No statistically significant differences in baseline demographics were noted between continent and incontinent patients (Table 4). Bivariate analyses showed that disease-specific quality of life was significantly lower and that daily activity impairment was significantly higher in incontinent patients compared with continent patients (Table 5). Specifically, incontinent OAB patients had an average I-QOL total score of 52, compared with 63

Table 5 Relationship between Quality of Life (QOL) and productivity measures and sncontinence status ${ }^{\mathrm{a}}$

\begin{tabular}{|c|c|c|c|c|}
\hline \multicolumn{3}{|c|}{ Variables } & \multicolumn{2}{|c|}{ Incontinence status $^{\mathrm{b}}$} \\
\hline & & & $\begin{array}{l}\text { Continent } \\
(\mathrm{n}=78)\end{array}$ & $\begin{array}{l}\text { Incontinent } \\
(\mathrm{n}=235)\end{array}$ \\
\hline \multirow[t]{3}{*}{ General QOL } & \multirow[t]{3}{*}{ EQ-5D utility score $\neq \neq$} & USA $^{*}$ & $0.82(0.22)$ & $0.68(0.24)$ \\
\hline & & Europe & $0.73(0.22)$ & $0.70(0.22)$ \\
\hline & & Total & $0.75(0.22)$ & $0.70(0.22)$ \\
\hline \multirow[t]{9}{*}{ Disease-specific QOL } & \multirow[t]{3}{*}{ I-QOL scorełキ } & USA $A^{* *}$ & $72(19)$ & $57(23)$ \\
\hline & & Europe $e^{* * *}$ & $59(17)$ & $50(19)$ \\
\hline & & Total*** & $63(19)$ & $52(20)$ \\
\hline & \multirow[t]{3}{*}{ OAB-q: Symptom severityłキ } & USA $^{*}$ & $37(19)$ & $49(19)$ \\
\hline & & Europe $e^{* * *}$ & $41(20)$ & $56(18)$ \\
\hline & & Total $\left.\right|^{* * *}$ & $40(20)$ & $54(19)$ \\
\hline & \multirow[t]{3}{*}{ 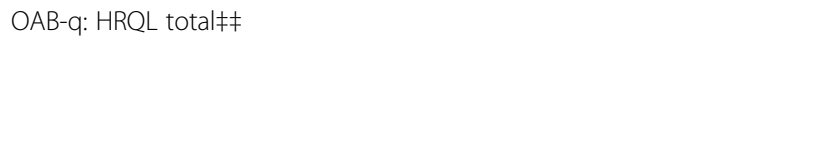 } & USA $A^{* * *}$ & $79(15)$ & $62(23)$ \\
\hline & & Europe & $63(19)$ & $57(19)$ \\
\hline & & Total** & $67(20)$ & $58(20)$ \\
\hline \multirow[t]{15}{*}{ Productivity } & \multirow[t]{3}{*}{ WPAl activity impairmentłキ } & USA & $34(26)$ & $46(28)$ \\
\hline & & Europe & $48(22)$ & $55(25)$ \\
\hline & & Total* & $44(24)$ & $52(26)$ \\
\hline & WPAI overall impairment & USA & $53(32)$ & $32(19)$ \\
\hline & \multirow{2}{*}{$\begin{array}{l}\text { (among patients on paid employment: } \mathrm{n} \text { (continent) }=19 \text {, } \\
\mathrm{n} \text { (incontinent) }=45) \neq \neq\end{array}$} & Europe & $49(22)$ & $50(28)$ \\
\hline & & Total & $50(23)$ & $43(26)$ \\
\hline & \multirow{3}{*}{$\begin{array}{l}\text { WPAl impairment while working (among patients on paid employment: } \\
\mathrm{n} \text { (continent) }=19, \mathrm{n} \text { (incontinent) }=45) \neq \neq\end{array}$} & USA & $38(33)$ & $33(18)$ \\
\hline & & Europe & $39(22)$ & $46(24)$ \\
\hline & & Total & $39(24)$ & $42(23)$ \\
\hline & \multirow{3}{*}{$\begin{array}{l}\text { WPAI work time miss (among patients on paid employment: } \\
\mathrm{n} \text { (continent) }=19, \mathrm{n} \text { (incontinent) }=45) \neq \neq\end{array}$} & USA & $15(26)$ & $1(2)$ \\
\hline & & Europe & $8(13)$ & $12(21)$ \\
\hline & & Total & $10(15)$ & $8(18)$ \\
\hline & \multirow[t]{3}{*}{ Proportion of patients on paid employment $\ddagger \Delta$} & USA & $5(23.8 \%)$ & $15(24.2 \%)$ \\
\hline & & Europe & $14(24.6 \%)$ & $30(17.9 \%)$ \\
\hline & & Total & 19 (24.4\%) & $45(19.6 \%)$ \\
\hline
\end{tabular}

${ }^{a}$ Bivariate analyses comparing burden of illness between incontinent versus non-incontinent group.

${ }^{b} 11$ out of 324 NDO patients had unknown incontinent status.

${ }^{*} \mathrm{p}<0.05{ }^{* *} \mathrm{p}<0.01{ }^{* * *} \mathrm{p}<0.001$ in chi-square test, Fisher's exact test, t-test, or Wilcoxon rank-sum test as appropriate.

$\neq$ Data are presented as number (percentage).

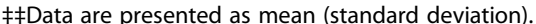

$\Delta$ Retired patients $(n=1)$ who reported "under paid employment" were analyzed as paid employees. 
in continent patients $(\mathrm{p}<0.001)$, In addition, incontinent patients had a significantly higher OAB-q symptom severity score and a lower OAB-q HRQoL score as compared to continent patients. Activity impairment, as assessed by the WPAI, was significantly higher in incontinent as compared with continent patients (52 vs 44 points, $\mathrm{p}<0.05$ ). Finally, in the US sample, the EQ-5D utility score was significantly lower in incontinent as compared to continent patients (0.68 vs. $0.82, \mathrm{p}<0.001)$. All of these differences were clinically significant (except for WPAI outcomes in which the MCIDs were relatively unclear), as defined by the published MCID of the respective instruments.

Slightly over $10 \%$ of the patients in the sample reported an OAB-related hospitalization in the past year, although there was no statistically significant difference in reported hospitalizations between continent and incontinent patients (Table 6). Similar rates were reported for OAB-related surgeries. On average, patients report 2 OAB-related office visits per year, with no statistically significant different between those who were continent compared to those who were incontinent. As expected, incontinence pad use was significantly higher among incontinent patients. On average, incontinent patients used 9.7 pads per week, compared with 1.8 pads per week for continent patients ( $\mathrm{p}<0.001)$. Reported hospitalization rates were higher for US compared to European patients.

After adjustment for age, sex, ethnicity, education level, insurance status, disease duration, country, and comorbidities, incontinence status remained significantly associated with lower disease-specific QOL and higher impairment in daily activities (Table 7). Compared with continent patients, incontinent patients on average scored 10 points lower on the I-QOL total score, 9 points lower on the OAB-q HRQoL score, 15 points higher on OAB-q symptom severity, and experienced $8.2 \%$ higher activity impairment due to their bladder condition (all $\mathrm{p}<0.05$ ). These differences were all clinically meaningful based on previously published MCIDs [13,20-23]. Sensitivity analyses confirmed these statistically significant and clinically meaningful relationships (Tables 7 and 8). Compared with continent patients, patients with more than 4 daily UI episodes had on average 14 points lower I-QOL total score, 12 points lower OAB-q HRQoL score, 20 points higher OAB-q symptom severity, and $14 \%$ higher activity impairment related to their bladder symptoms (all p <0.001) (Table 7). Although general HRQoL was not statistically significantly different between incontinent and continent patients, patients with more

Table 6 Relationship between health resource utilization and incontinence status ${ }^{a}$

\begin{tabular}{|c|c|c|c|}
\hline \multicolumn{2}{|l|}{ Variables } & \multicolumn{2}{|c|}{ Incontinence status $^{\mathrm{b}}$} \\
\hline & & \multirow{2}{*}{$\begin{array}{c}\begin{array}{c}\text { Continent } \\
(\mathbf{n}=\mathbf{7 8})\end{array} \\
1(5.0 \%)\end{array}$} & \multirow{2}{*}{$\begin{array}{c}\text { Incontinent } \\
\text { (n = 235) }\end{array}$} \\
\hline Proportion of patients having OAB-related hospitalization experience during the past 12 months & USA & & \\
\hline & Europe & $9(15.8 \%)$ & $31(18.2 \%)$ \\
\hline & Total & $10(12.8 \%)$ & $34(14.5 \%)$ \\
\hline \multirow[t]{3}{*}{ Proportion of patients that ever had OAB-related surgery $\neq$} & USA & $2(9.5 \%)$ & $10(15.9 \%)$ \\
\hline & Europe & $6(10.5 \%)$ & $11(6.5 \%)$ \\
\hline & Total & $8(10.3 \%)$ & $21(9.1 \%)$ \\
\hline \multirow[t]{3}{*}{ Proportion of patients that had switched anticholinergics used for $O A B$ due to efficacy/side effectsł } & USA & $6(28.6 \%)$ & $15(23.1 \%)$ \\
\hline & Europe & $18(31.6 \%)$ & $46(27.1 \%)$ \\
\hline & Total & $24(30.8 \%)$ & $61(26.0 \%)$ \\
\hline \multirow[t]{3}{*}{ Proportion of patients currently using pads (at the time of completing the survey) $\neq$} & USA** & $4(19.0 \%)$ & $40(61.5 \%)$ \\
\hline & Europe $e^{* * *}$ & $6(10.5 \%)$ & $127(75.6 \%)$ \\
\hline & Total ${ }^{* * *}$ & $10(12.8 \%)$ & $167(71.7 \%)$ \\
\hline \multirow[t]{3}{*}{ Number of OAB-related physician visits over the past 3 monthsł‡ } & USA & $1.3(1.2)$ & $1.5(1.3)$ \\
\hline & Europe & $2.2(2.4)$ & $2.5(2.3)$ \\
\hline & Total & $2.0(2.1)$ & $2.2(2.0)$ \\
\hline \multirow[t]{3}{*}{ 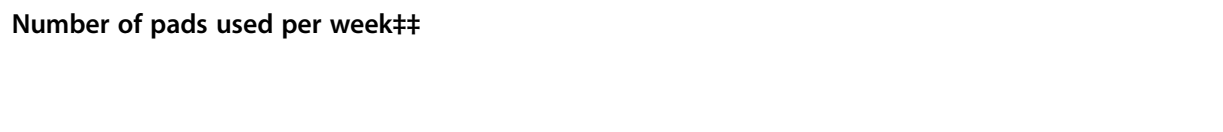 } & USA & $4.5(12.2)$ & $10.8(14.4)$ \\
\hline & Europe $e^{* * *}$ & $0.8(2.4)$ & $9.3(9.0)$ \\
\hline & Total*** & $1.8(6.7)$ & $9.7(10.8)$ \\
\hline
\end{tabular}

${ }^{\mathrm{a}}$ Bivariate analyses comparing burden of illness between incontinent versus non-incontinent group.

${ }^{\mathrm{b}} 11$ out of 324 NDO patients had unknown incontinent status.

${ }^{*} \mathrm{p}<0.05{ }^{* *} \mathrm{p}<0.01{ }^{* * *} \mathrm{p}<0.001$ in chi-square test, Fisher's exact test, t-test, or Wilcoxon rank-sum test as appropriate.

$\neq$ Data are presented as number (percentage).

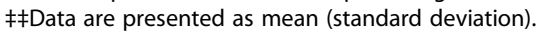


Table 7 Primary analysis: adjusted associations between incontinence status and quality of life (QOL)/productivity measures using linear regression models $(n=324)^{\dagger}$

\begin{tabular}{|c|c|c|c|c|c|c|c|}
\hline \multirow[t]{3}{*}{ Quality of life outcomes } & \multicolumn{7}{|c|}{ Incontinence status } \\
\hline & \multicolumn{2}{|c|}{ Base-case analysis } & \multicolumn{5}{|c|}{ Sensitivity analysis } \\
\hline & $\begin{array}{l}\text { Incontinence } \\
\text { (Yes vs. No) }\end{array}$ & $\mathrm{R}^{2}$ & $\begin{array}{l}\text { Average number of UI } \\
\text { episodes per day }\end{array}$ & $\mathrm{R}^{2}$ & $\begin{array}{r}\text { Nun } \\
\text { episod }\end{array}$ & $\begin{array}{l}\text { nber of UI } \\
\text { les (Ordinal) }\end{array}$ & $\mathrm{R}^{2}$ \\
\hline \multirow[t]{2}{*}{ EQ-5D utility score ${ }^{a}$} & $-0.04(0.03)$ & 0.21 & $-0.01(0.00)^{*}$ & 0.22 & $0 \leq n \leq 4$ & $-0.02(0.03)$ & 0.22 \\
\hline & & & & & $n>4$ & $-0.07(0.03)^{*}$ & \\
\hline \multirow[t]{2}{*}{ I-QOL score ${ }^{a}$} & $-10.39(2.52)^{* * *}$ & 0.25 & $-1.03(0.29)^{* * *}$ & 0.24 & $0 \leq n \leq 4$ & $-8.47(2.69)^{* *}$ & 0.26 \\
\hline & & & & & $n>4$ & $-13.66(2.97)^{* * *}$ & \\
\hline \multirow[t]{2}{*}{ OAB-q symptom severity score ${ }^{a}$} & $15.01(2.58)^{* * *}$ & 0.22 & $1.65(0.29)^{* * *}$ & 0.22 & $0 \leq n \leq 4$ & $11.92(2.72)^{* * *}$ & 0.25 \\
\hline & & & & & $\mathrm{n}>4$ & $20.27(3.01)^{* * *}$ & \\
\hline \multirow[t]{2}{*}{ OAB-q HRQL total score ${ }^{a}$} & $-8.69(2.72)^{* *}$ & 0.19 & $-1.03(0.30)^{* * *}$ & 0.19 & $0 \leq n \leq 4$ & $-6.63(2.89)^{*}$ & 0.20 \\
\hline & & & & & $\mathrm{n}>4$ & $-12.22(3.18)^{* * *}$ & \\
\hline \multirow[t]{2}{*}{ WPAI - percent activity impairment due to problem ${ }^{a}$} & $8.23(3.41)^{*}$ & 0.15 & $1.40(0.38)^{* * *}$ & 0.17 & $0 \leq n \leq 4$ & $4.88(3.62)$ & 0.17 \\
\hline & & & & & $n>4$ & $13.95(4.03)^{* *}$ & \\
\hline \multirow[t]{2}{*}{ WPAI - percent work time miss due to problem $\ddagger^{\mathrm{a}, \mathrm{b}}$} & $3.69(5.66)$ & 0.22 & $1.51(1.15)$ & 0.24 & $0 \leq n \leq 4$ & $3.51(5.90)$ & 0.22 \\
\hline & & & & & $n>4$ & $4.71(8.98)$ & \\
\hline \multirow{2}{*}{$\begin{array}{l}\text { WPAI - percent impairment while working } \\
\text { due to problem } \neq^{\mathrm{a}, \mathrm{b}}\end{array}$} & $3.70(7.13)$ & 0.28 & $3.14(1.42)^{*}$ & 0.34 & $0 \leq n \leq 4$ & $1.69(7.16)$ & 0.31 \\
\hline & & & & & $n>4$ & $15.93(11.33)$ & \\
\hline \multirow{2}{*}{$\begin{array}{l}\text { WPAI - percent overall work impairment due to } \\
\text { problem } \ddagger^{a, b}\end{array}$} & $3.04(8.96)$ & 0.27 & $1.94(1.79)$ & 0.28 & $0 \leq n \leq 4$ & $3.30(9.30)$ & 0.27 \\
\hline & & & & & $n>4$ & $1.21(13.88)$ & \\
\hline
\end{tabular}

†All analyses adjusted for time since diagnosis, age, male sex, race, education, insurance status, country, and whether having the following comorbidities: psychological/psychiatric conditions, hypertension, diabetes, and dementia. ‡Comorbidities were not included as covariates.

${ }^{a}$ Mean regression coefficient (non-exponentiated) (standard error).

${ }^{\mathrm{b}}$ Population restricted to patients that were on paid employment $(n=68)$.

'Reference group: patients that reported no leakages.

${ }^{*} p<0.05 * * p<0.01 * * * p<0.001$.

than 4 daily UI episodes (compared with patients with no leakages) on average had 0.07 lower EQ-5D utility score $(\mathrm{p}<0.05)$ (Table 7). Additionally, number of UI episodes was statistically significantly associated with lower EQ-5D utility scores: one additional episode was associated with a 0.01 point decrease in utility $(\mathrm{p}<0.05)$. While qualitative differences were observed in work productivity impairment, these differences were not statistically significant.

\section{Discussion}

In the current study, incontinent patients with an underlying neurological condition were found to have significantly lower disease-specific HRQoL and greater activity impairment compared with continent patients. However, the differences in general HRQoL and work productivity were not statistically significant. One potential explanation is that the severity of the patients' underlying neurological disease may limit the ability to distinguish the incremental impact of their urinary incontinence. Secondly, it is possible that the EQ-5D may lack the sensitivity to detect such differences. Thirdly, the study was not specifically powered to observe such differences, particularly in the case of the WPAI. All analyses on absenteeism, presenteeism, and overall work impairment included only self-identified paid employees $(n=66)$. In general, patients with NDO may have a lower employment rate due to their underlying neurological conditions. In this analysis, the proportion of NDO patients on paid employment was significantly lower as compared to other OAB patients $(21 \%$ vs. $36 \%, \mathrm{p}<0.001)$. Further investigations to verify these associations are recommended on a larger sample of NDO patients.

The current study is, to our knowledge, the first study that systematically evaluates the HRQoL, productivity burden, and healthcare resource utilization between incontinent and continent NDO patients. Previous investigations focused on estimating the burden of illness in the general $\mathrm{OAB}$ population and identified significant relationships between incontinence and both general and disease-specific quality of life. Specifically, Coyne et al. found that both men and women with incontinent $\mathrm{OAB}$ were significantly more likely to report $\mathrm{OAB}$ symptom-specific bother (odds ratio $(\mathrm{OR})=1.81$ in men and 1.92 in women, respectively) [10]. In another investigation by Coyne and colleagues, significantly lower 
Table 8 Sensitivity analysis: adjusted associations between incontinence status and quality of life (QOL)/ productivity measures using negative binomial regression models $(n=324)^{\dagger}$

\begin{tabular}{|c|c|c|c|c|}
\hline \multirow{4}{*}{$\begin{array}{l}\text { Quality of life outcomes } \\
\text { EQ-5D utility score }\end{array}$} & \multicolumn{4}{|c|}{ Incontinence status } \\
\hline & \multirow{3}{*}{$\begin{array}{c}\text { Base-case analysis } \\
\text { Incontinence } \\
\text { (Yes vs. No) }\end{array}$} & \multicolumn{3}{|c|}{ Sensitivity analysis } \\
\hline & & \multirow{2}{*}{$\begin{array}{c}\begin{array}{c}\text { Average number of UI } \\
\text { episodes per day }\end{array} \\
0.99(0.02)\end{array}$} & \multicolumn{2}{|c|}{$\begin{array}{c}\text { Number of UI } \\
\text { episodes (Ordinal) }\end{array}$} \\
\hline & & & $0 \leq n \leq 4$ & $0.97(0.16)$ \\
\hline & & & $\mathrm{n}>4$ & $0.91(0.17)$ \\
\hline \multirow[t]{2}{*}{ I-QOL score ${ }^{a}$} & $0.83(0.04)^{* * *}$ & $0.98(0.01)^{* *}$ & $0 \leq n \leq 4$ & $0.86(0.05)^{* *}$ \\
\hline & & & $\mathrm{n}>4$ & $0.78(0.05)^{* * *}$ \\
\hline \multirow[t]{2}{*}{ OAB-q symptom severity score ${ }^{a}$} & $1.38(0.08)^{* * *}$ & $1.03(0.01)^{* * *}$ & $0 \leq n \leq 4$ & $1.30(0.08)^{* * *}$ \\
\hline & & & $\mathrm{n}>4$ & $1.52(0.10)^{* * *}$ \\
\hline \multirow[t]{2}{*}{ OAB-q HRQL total score ${ }^{a}$} & $0.87(0.05)^{* *}$ & $0.98(0.01)^{* *}$ & $0 \leq n \leq 4$ & $0.90(0.05)$ \\
\hline & & & $\mathrm{n}>4$ & $0.81(0.05)^{* *}$ \\
\hline \multirow{2}{*}{$\begin{array}{l}\text { WPAI - percent activity impairment due } \\
\text { to problem }\end{array}$} & $1.21(0.10)^{*}$ & $1.03(0.01)^{* *}$ & $0 \leq \mathrm{n} \leq 4$ & $1.12(0.10)$ \\
\hline & & & $\mathrm{n}>4$ & $1.34(0.13)^{* *}$ \\
\hline \multirow[t]{2}{*}{ WPAI - percent work time miss due to problem $\ddagger^{\mathrm{a}, \mathrm{b}}$} & $2.77(3.11)$ & $1.46(0.48)$ & $0 \leq n \leq 4$ & $2.89(3.29)$ \\
\hline & & & $\mathrm{n}>4$ & $1.87(3.86)$ \\
\hline \multirow{2}{*}{$\begin{array}{l}\text { WPAI - percent impairment while working due } \\
\text { to problem } \neq^{a, b}\end{array}$} & $1.04(0.26)$ & $1.08(0.05)$ & $0 \leq \mathrm{n} \leq 4$ & $0.99(0.25)$ \\
\hline & & & $\mathrm{n}>4$ & $1.39(0.52)$ \\
\hline \multirow{2}{*}{$\begin{array}{l}\text { WPAI - percent overall work impairment due } \\
\text { to problem } \neq^{\mathrm{a}, \mathrm{b}}\end{array}$} & $1.03(0.39)$ & $1.02(0.07)$ & $0 \leq n \leq 4$ & $1.06(0.40)$ \\
\hline & & & $n>4$ & $0.81(0.45)$ \\
\hline
\end{tabular}

tAll analyses adjusted for time since diagnosis, age, male sex, race, education, insurance status, country, and whether having the following comorbidities: psychological/psychiatric conditions, hypertension, diabetes, and dementia. $\neq$ Comorbidities were not included as covariates.

ancidence rate ratio (standard error).

${ }^{\mathrm{b}}$ Population restricted to patients that were on paid employment $(n=68)$.

'Reference group: patients that reported no leakages.

${ }^{*} p<0.05 * * p<0.01{ }^{* * *} p<0.001$.

utility score and greater bother in all subscales of OAB-q was detected in incontinent $\mathrm{OAB}$ patients as compared to continent OAB patients [9]. By estimating SF-36 domain scores, Stewart et al. found clinically significant differences in 7 out of 8 domain scores between incontinent and continent patients [8]. In the current study, general quality of life as assessed by the EQ-5D did not significantly differ in the NDO patients. As previously mentioned, the total burden of illness in neurogenic patients includes the neurologic and urinary condition, along with any additional comorbidities. Thus, the impact of urinary incontinence itself may be difficult to ascertain among these patients. Furthermore, the sensitivity of the EQ-5D $3 \mathrm{~L}$ may be limited to assess such differences, in contrast to the diseasespecific instruments. The sensitivity of alternative utility instruments such as the OAB-5D, developed specifically for an overactive bladder population from the OAB-q, may be worth exploring in future studies [26]. In addition, as the current study focuses on the incremental impact of urinary incontinence (as opposed to frequency and other symptoms of $\mathrm{OAB}$ ), a utility measure developed from an incontinence-specific instrument such as the I-QOL may be another viable alternative.
Major strengths of this study included the direct specification of patient diagnoses from their physicians, the strict inclusion criteria applied during patient recruitment, and the exclusion of mixed-type incontinent patients. Moreover, the effect of recall bias on the frequency of incontinence episodes was relatively low, as it was determined using a recall period of one week as compared to four weeks in previous investigations.

The current study was also subjected to limitations. The survey data were collected using non-random sampling methods, and the strict inclusion criteria for the subsequent analysis may limit the generalizability of results to those with similar characteristics as the study population. Secondly, causal relationships between incontinence status and quality of life cannot be verified due to the cross-sectional nature of the survey data. Thirdly, the MCID value pertaining to EQ-5D $3 \mathrm{~L}$ was based on chronic disease patients other than $\mathrm{OAB}$ patients. Ideally, future research might validate the instrument in the $\mathrm{OAB}$ population, particularly among patients with a neurological condition. Finally, statistical correlations among patients recruited by the same physicians may potentially influence the study results. 


\section{Conclusion}

The current investigation found that in NDO patients in Western Europe and the US, urinary incontinence was associated with clinically and statistically lower disease-specific QOL and greater daily activity impairment. These associations were robust as demonstrated by the sensitivity analyses. These results suggest that-in addition to the patients' underlying neurological conditions, their urinary incontinence contributes to a significant degree of humanistic burden. Healthcare providers may consider such impacts on the patients' quality of life when selecting appropriate treatments.

\section{Abbreviations}

NDO: Neurogenic detrusor overactivity; UI: Urinary incontinence;

HRQoL: Health-related quality of life; HRU: Health resource use;

OAB: Overactive bladder; EQ-5D: EuroQoL-5D; I-QOL: Incontinence Quality of

Life questionnaire; OAB-q: Overactive Bladder Questionnaire; WPAl: Work

Productivity and Activity Impairment; OLS: Ordinary least squares;

MCID: Minimal clinically important differences; SD: Standard deviation;

MS: Multiple sclerosis; SCl: Spinal cord injury; PD: Parkinson's disease;

DSP: Disease-Specific Programmes; PRF: Patient record form; PSC: Patient self-completion; EQ-5D 3 L: EuroQoL-5D 3 level; VAS: Visual analog scale; OR: Odds ratio.

\section{Competing interests}

Throughout the study completion process, Derek H Tang, MS, PhD Candidate, served as a paid intern for the department of Global Health Outcomes Strategy and Research, Allergan Inc.; Danielle Colayco, PharmD, MS, served as a full-time employee, followed by transition into a paid consultant for the department of Global Health Outcomes Strategy and Research, Allergan Inc.; Denise Globe, PhD, and Vaishali Patel, PharmD, MS, have been full-time employees of the department of Global Health Outcomes Strategy and Research, Allergan Inc.; Michael B Chancellor, MD, served as a consultant and investigator with Allergan Inc. The descriptions above refer specifically to the approaches and analysis for this specific manuscript to address the research questions of quality of life and utilization between those with and without incontinence; the actual broader study (i.e., the process of creating the Adelphi OAB/UI data set) was developed and fielded by Adelphi Values with input from Vaishali Patel and Tina Chiang from Allergan Inc.

\section{Authors' contribution}

DHT made substantial contributions to the study design, analysis, interpretation of data, and drafting the manuscript; DC, DG, and VP made substantial contributions to the study design; DC, DG, VP, JP, and MBC made critical revision of the manuscript for important intellectual content. All authors have read and approved the final version of the manuscript.

\section{Authors' information}

At the time of the analysis for this paper, Danielle Colayco was an employee of Allergan, Inc.

\section{Acknowledgements}

We would like to acknowledge Dr. Kristin Khalaf, PharmD, MS, for providing inputs to solidify the study design. This research was not funded in addition to what has been described in the Competing Interests section.

\section{Author details}

1The University of Arizona College of Pharmacy, 1295 N. Martin, PO Box 210202, Tucson, AZ 85721, USA. ²Department of Global Health Outcomes Strategy and Research, Allergan Inc, 2525 Dupont Drive, Irvine, CA 92612, USA. ${ }^{3}$ Adelphi Real World, Adelphi Mill, Grimshaw Lane, Bollington SK10 5JB, UK. ${ }^{4}$ Oakland University William Beaumont School of Medicine, Neurourology Program, Beaumont Hospital, 3535 West 13 Mile Road, Royal Oak, Ml 48073, USA.
Received: 4 January 2013 Accepted: 20 March 2014

Published: 4 April 2014

\section{References}

1. Abrams P, Cardozo L, Fall M, Griffiths D, Rosier P, Ulmsten U, van Kerrebroeck $P$, Victor A, Wein A: The standardization of terminology of lower urinary tract function: report from the standardisation sub-committee of the International Continence Society. Neurourol Urodyn 2002, 21:167-178.

2. Ganz ML, Smalarz AM, Krupski TL, Anger JT, Hu JC, Wittrup-Jensen KU, Pashos CL: Economic costs of overactive bladder in the United States. Urology 2010, 75(3):526-532. 532 e1-18.

3. Reeves P, Irwin D, Kelleher C, Milsom I, Kopp Z, Calvert N, Lloyd A: The current and future burden and cost of overactive bladder in five European countries. Eur Urol 2006, 50:1050-1057.

4. Coyne KS, Sexton CC, Irwin DE, Kopp ZS, Kelleher CJ, Milsom I: The impact of overactive bladder, incontinence and other lower urinary tract symptoms on quality of life, work productivity, sexuality and emotional well-being in men and women: results from the EPIC study. BJU Int 2008, 101:1388-1395

5. Asche CV, Kim J, Kulkarni AS, Chakravarti P, Andersson K: Presence of central nervous system, cardiovascular and overall co-morbidity burden in patients with overactive bladder disorder in a real-world setting. BJU Int 2011, 109:572-580.

6. Denys $P$, Schnitzler A, Roche N: Managing patients with neurogenic detrusor overactivity-a global approach. Eur Urol Suppl 2006, 5:691-695.

7. Fowler CJ, Panicker JN, Drake M, Harris C, Harrison SCW, Kirby M, Lucas M, Macleod N, Mangnall J, North A, Porter B, Reid S, Russell N, Watkiss K, Wells M: A UK consensus on the management of the bladder in multiple sclerosis. J Neurol Neurosurg Psychiatry 2009, 80:470-477.

8. Stewart WF, van Rooyen JB, Cundiff GW, Abrams P, Herzog AR, Corey R, Hunt TL, Wein AJ: Prevalence and burden of overactive bladder in the United States. World J Urol 2003, 20:327-336.

9. Coyne KS, Payne C, Bhattacharyya SK, Revicki DA, Thompson C, Corey R, Hunt TL: The impact of urinary urgency and frequency on health-related quality of life in overactive bladder: results from a national community survey. Value Health 2004, 7:455-463.

10. Coyne KS, Sexton CC, Kopp ZS, Ebel-Bitoun C, Milsom I, Chapple C: The impact of overactive bladder on mental health, work productivity and health-related quality of life in the UK and Sweden: results from EpiLUTS. BJU Int 2011, 108:1459-1471.

11. Anderson P, Benford M, Harris N, Karavali M, Piercy J: Real-world physician and patient behaviour across countries: disease-specific programmes-a means to understand. Curr Med Res Opin 2008, 24:3063-3072.

12. Brazier J, Jones N, Kind P: Testing the validity of the EuroQol and comparing it with the SF-36 health survey questionnaire. Qual Life Res 1993, 2:169-180.

13. Schurch B, Denys P, Kozma CM, Reese PR, Slaton T, Barron R: Reliability and validity of the incontinence quality of life questionnaire in patients with neurogenic urinary incontinence. Arch Phys Med Rehabil 2007, 88:646-652.

14. Coyne K, Revicki D, Hunt T, Corey R, Stewart W, Bentkover J, Kurth H, Abrams P: Psychometric validation of an overactive bladder symptom and health-related quality of life questionnaire: the OAB-q. Qual Life Res 2002, 11(6):563-574.

15. Reilly MC, Zbrozek AS, Dukes EM: The validity and reproducibility of a work productivity and activity impairment instrument. Pharmaecoconomics 1993, 4:353-365.

16. Calculating the U.S. Population-based EQ-5D ${ }^{\mathrm{TM}}$ Index Score. In Rockville, MD, USA: Agency for Healthcare Research and Quality; 2005. [http://www. ahrq.gov/rice/EQ5Dscore.htm]

17. Patient Reported Outcomes in Lower Urinary Tract Symptoms (LUTS) and Sexual Health: Overactive Bladder Questionnaires. In New York, NY, USA: Pfizer; 2008. [http://www.oabq.org]

18. Incontinence Quality of Life Questionnaire. In. Vancouver, BC, CAN: Spinal Cord Injury Rehabilitation Evidence; 2010. [http://www.scireproject.com/ outcome-measures/incontinence-quality-of-life-questionnaire-i-qol]

19. WPAl scoring. In. New York, NY, USA: Reilly Associates; 2011. [http://www.reillyassociates.net/WPAl_Scoring.html]

20. Walters SJ, Brazier JE: Comparison of the minimally important difference for two health state utility measures: EQ-5D and SF-6D. Qual Life Res 2005, 14:1523-1532 
21. Luo N, Johnson JA, Shaw JW, Coons SJ: Relative efficiency of the EQ-5D, $\mathrm{HUI} 2$, and HUI3 index scores in measuring health burden of chronic medical conditions in a population health survey in the United States. Med Care 2009, 47(1):53-60.

22. Coyne KS, Matza LS, Thompson CL, Kopp ZS, Khullar V: Determining the importance of change in the overactive bladder questionnaire. J Urol 2006, 176:627-632.

23. Dyer K, Xu Y, Brubaker L, Nygaard I, Markland A, Rahn D, Chai TC, Stoddard A, Lukacz E: Minimum important difference for validated instruments in women with urge incontinence. Neurourol Urodynam 2011, 30:1319-1324.

24. Bolge SC, Doan JF, Kannan H, Baran RW: Association of insomnia with quality of life, work productivity, and activity impairment. Qual Life Res 2009, 18:415-422.

25. Reilly MC, Brown M, Brabant Y, Gerlier L, Sandborn WJ: Defining the minimally important difference of WPAI:CDAl scores: what is a relevant impact on work productivity in active Crohn's disease? [abstract]. Gut 2007, 56(Suppl. III):A159.

26. Yang Y, Brazier J, Tsuchiya A, Coyne K: Estimating a preference-based single index from the Overactive Bladder questionnaire (OAB-q). Value Health 2009, 12(1):159-166.

doi:10.1186/1471-2377-14-74

Cite this article as: Tang et al:: Impact of urinary incontinence on health-related quality of life, daily activities, and healthcare resource utilization in patients with neurogenic detrusor overactivity. BMC Neurology 2014 14:74

\section{Submit your next manuscript to BioMed Central and take full advantage of:}

- Convenient online submission

- Thorough peer review

- No space constraints or color figure charges

- Immediate publication on acceptance

- Inclusion in PubMed, CAS, Scopus and Google Scholar

- Research which is freely available for redistribution 